\title{
Developing a Smart Device for Measuring and Monitoring Ammonia Concentration
}

\author{
BUI Dang Thanh*, PHAM Van Truong, NGUYEN Huy Phuong \\ Hanoi University of Science and Technology - No. 1, Dai Co Viet str., Hai Ba Trung dist., Ha Noi, Viet Nam \\ Received: June 09, 2020; Accepted: November 12, 2020
}

\begin{abstract}
This paper presents an approach for design and realization of a smart device for measuring and monitoring ammonia concentration. The developed system included two parts: hardware and software, in which the hardware of the system has been developed based on the ATMEGA328P microcontroller in order to collect data from the ammonia sensor MQ135. Meanwhile, the software is developed for signal processing and warning according to different thresholds. Collected data by the system is then transmitted to a computer via the wireless communication protocol. In addition, an interface is also designed on computers to collect and monitor data from sensors. The developed system allows to measure ammonia concentration up to 300ppm and has been tested not only at the laboratory but also at NPK factory of Lamthao Fertilizers and Chemicals Joint Stock Company. Experimental results show the desired performances of the developed system.
\end{abstract}

Keywords: Ammonia concentration, MCU, smart device, wireless communication

\section{Introduction}

NPK is a fertilizer that fertilizer provides many important nutrients, to make plants lusher or grow height and volume of leaf stems, form fruit, sprout buds... Nowadays, high tower technology is the main technics for NPK fertilizer production. This is a modern fertilizer production technology applied in many countries. Raw materials for fertilizer production such as $\left(\mathrm{NH}_{2}\right)_{2} \mathrm{CO}, \mathrm{NH}_{4} \mathrm{H}_{2} \mathrm{PO}_{4}, \mathrm{KNO}_{3}, \mathrm{CaCO}_{3}$ and other trace elements are mixed, and to be taken to NPK granulation tower. At this time, heating and maintaining the materials at a certain temperature will melt down the main material, Urea $\left(\left(\mathrm{NH}_{2}\right)_{2} \mathrm{CO}\right)$, mixing it with other materials to form an almost homogeneous fluid. After that, this fluid will be automatically discharged to a centrifugal granulator. It will form fluid particles free-falling in the center of the tower. Meanwhile, an ultra-high-speed blower system will blow cool and strong airflow from the bottom of the tower to reduce falling speed of the particles as well as dry them. The dry and round particles will slowly fall to the separator sieve of product classification. And from that equipment, the qualified particles will be forwarded to the system of anti-clumping membrane spraying. The last step is packing and delivering to customers.

During NPK fertilizer manufacturing process, the factory generates poison emissions into natural environment and working environment, which is seriously affecting the environment as well as human health. In the poison gases generated, ammonia is a gas that causes many harms to human health. In more detail, these nanosized ammonia aerosols create smog that exhibits a temperature reducing effect and, consequently, a negative impact on the global greenhouse balance [1, 2]. In addition, long-term exposure to ammonia at an unsafe level could cause pulmonary and lung diseases to humans and animals besides other health issues [3, 4]. Therefore, it is necessary to develop devices that can measure and monitor the parameters of ammonia.

A vast number of researches related to this field have been proposed in the literature. Some studies focused on developing sensors to measure ammonia concentrations as the work in [5]. Other studies focus on designing and optimizing sensors based on titanium oxide nanowire for measuring gas concentration [6]. In [7], the authors studied the operating principle of the sensor based on the change in work function of a sensitive film due to gas adsorption on its surface. This sensor was used for measuring ammonia concentration in room temperature. In $[8,9]$, the authors used ceramic materials as inorganic oxide semiconductors to made gas sensor. Arshak et al. in [10] used thin film-based metal oxides assensing materials saturate quickly to product gas sensor. In another approach, conducting polymers are also used as sensing layers in gas sensors. The advantages of the use of polymers as sensing layers are that they allow room temperature operation,

\footnotetext{
* Corresponding author: Tel.: +84 02438683518

Email: thanh.buidang@hust.edu.vn
} 
and feature a high sensing response, short response time, and ease of device fabrication $[11,12]$. On the other hand, recently, microcontrollers and embedded computers have been applied in the ammonia measured and monitored devices. The main advantage of using microcontrollers and embedded computers is to make these devices more intelligent and flexible [13]. The authors in [14] used available module to create the measurement system. This system has no communication part, no data acquisition part, and no data display part on the webserver.

From the above analysis, in this study, we develop a smart device with smart warning based on multiple thresholds and wireless connection using microcontroller to measure and monitor ammonia concentration. In more detail, in the current work, we developed a sensor based on conductive polymer. The developed system is then applied for NPK fertilizer industry and reveals a good sensing response that increases considerably with the addition of $\mathrm{N}$-doped graphene quantum dots (N-GQDs). It is noted that, compared to the work in [14], in this work, we developed a device that can connect to an IoT system. Thus, the range of applying in chemistry industrial of the device is reasonable and available.

\section{Ammonia Concentration Measurement Method}

\subsection{Electrochemical Method}

This method works by allowing gas to diffuse through a porous membrane to the electrode. The oxidized or reduced electrode leads to the amount of generated electricity determined by the oxidized gas at the electrode, thereby determining the gas concentration. This sensor has been used in various environments such as oil refineries, gas turbines, chemical plants,... The electrochemical method has some advantages, such as high stability, fast speed, and less maintenance. However, in this method, sensors are subjected to corrosion or chemical contamination, and lifespan is only from 1 to 2 years.

\subsection{Semiconductor Method}

The principle of this method bases on chemical reactions, i.e., when sensor is in direct contact with gas, the resistor of the sensor will reduce in proportion to the exposure gas concentration. Resistors in semiconductor sensors are mainly made from tin dioxide (about $50 \mathrm{k} \Omega$ in air, can be reduced to about $3.5 \mathrm{k} \Omega$ when there is $1 \%$ methane). Semiconductor sensors are often used to detect hydrogen, oxygen, alcohol vapor, and toxic gases such as carbon monoxide [15]. The advantages of semiconductor method can be listed as high stability, fast speed, less maintenance, and low price. Nevertheless, sensors are subjected to corrosion or chemical contamination.

\subsection{Using MQ135 Sensor to Determine Ammonia Concentration}

\subsubsection{MQ135 Sensor}

MQ135, a gas sensor manufactured by Hanwei Sensor from Taiwan, is based on the principle of conductivity (semiconductor sensor). The material of sensor is Tin oxide $\left(\mathrm{SnO}_{2}\right)$ with low electrical conductivity in clean air. The principle is about detecting the change in conductivity of thin-membrane semiconductors when absorbing gas on the surface at high temperatures. When the sensor detects gas, the resistor of the Tin oxide layer decreases in proportion to the gas concentration. This sensor is mainly used to measure toxic gases such as $\mathrm{NOx}, \mathrm{NH}_{3}, \mathrm{CO}, \ldots$ The specifications of MQ135 are listed as:

- Voltage of the burner: $5 \mathrm{~V} \pm 0.1 \mathrm{AC} / \mathrm{DC}$;

- Load resistor: Changeable $(2 \mathrm{k} \Omega-47 \mathrm{k} \Omega)$;

- Resistor of the heating unit: $33 \Omega \pm 5 \%$;

- Detection range: up to $300 \mathrm{ppm}$ ammonia.

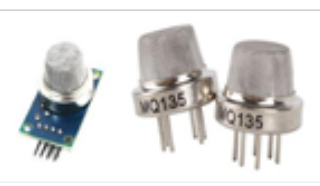

a) MQ 135 Sensor Module

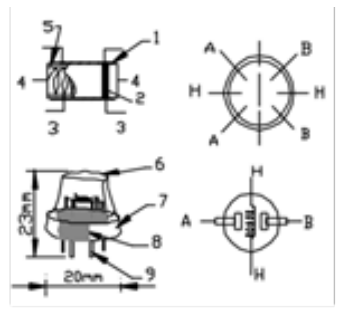

b) Struture of MO 135 Sensor
Fig. 1. MQ135 sensor

Description of signal pin reference of MQ135 sensor structure is shown in Table 1.

Table 1. Reference for Structure of MQ125 Sensor

\begin{tabular}{|c|c|c|}
\hline No. & Name & Material \\
\hline 1 & Thin- membrane & $\mathrm{SnO}_{2}$ \\
\hline 2 & Electrode & $\mathrm{Au}$ \\
\hline 3 & Wire & $\mathrm{Pt}$ \\
\hline 4 & Incandescent & $\mathrm{Ni}-\mathrm{Cr}$ alloy \\
\hline 5 & Cylindrical block & $\mathrm{Al}_{2} \mathrm{O}_{3}$ \\
\hline 6 & Protective nets & Stainless steel \\
\hline 7 & Clamp ring & Ni copper plating \\
\hline 8 & Plastic soles & Bakelite \\
\hline 9 & PIN pins & Ni copper plating \\
\hline
\end{tabular}

The sensor is supplied with two voltage sources: the calcined voltage $\left(V_{H}\right)$ and the supply voltage $\left(V_{C}\right)$. The source used to heat the burner, $V_{C}$ used to generate voltage $\left(V_{\text {out }}\right)$ on the load resistor $\left(R_{L}\right)$. Two sources $V_{C}$ and $V_{H}$ can use the same power circuit to ensure sensor performance. To use the sensor with the 
best performance, the appropriate value for $R_{L}$ is $20 k \Omega$ (adjustment range from $20 k \Omega$ to $47 k \Omega$ ).

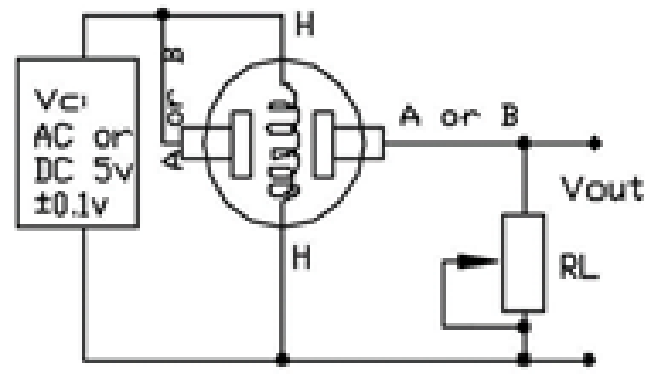

Fig. 2. Principle diagram

Considering environmental conditions: temperature $20^{\circ} \mathrm{C}$, humidity $65 \%, \mathrm{O}_{2}$ concentration $21 \%$ and load resistor $R_{L}=20 \mathrm{k} \Omega$.

The sensor capacity and sensor resistor can be calculated as:

$$
\begin{aligned}
& P_{S}=V_{C}^{2} \frac{R_{S}}{\left(R_{S}+R_{L}\right)^{2}} \\
& R_{S}=\left(\frac{V_{C}}{V_{\text {out }}}-1\right) R_{L}
\end{aligned}
$$

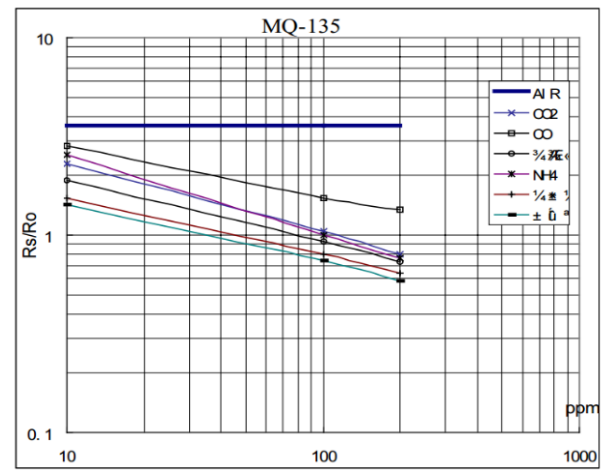

Fig. 3. Graph of characteristics of MQ135

It is noted that: In Figure 2, $R_{L}$ combines with resistor of sensor $\left(R_{A B}\right)$ to form a voltage circuit. The voltage on $R_{L}$ is proportional to the gas concentration received by the sensor. In Figure $3, R_{0}$ is the value of the sensor's resistor at $100 \mathrm{ppm}$ ammonia, and $R_{S}$ is the value of the sensor's resistor at other conditions of the air.

The MQ135 sensor is affected by temperature and humidity, so when surveyed, the temperature and humidity of the corresponding environment should be determined for each measured value.

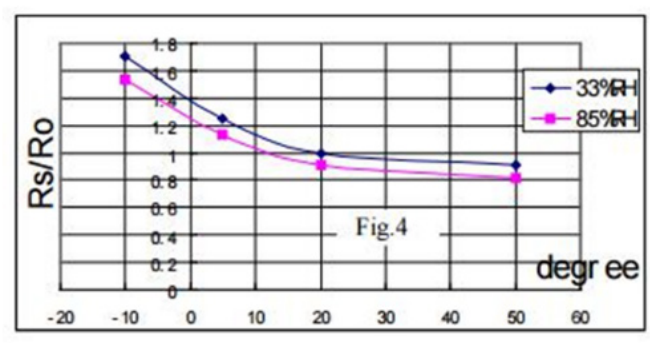

Fig. 4. Effect of temperature and humidity to MQ135

\subsubsection{Develop ammonia Equation for MQ135}

The first step is to find the resistor value $R_{0}$. From Figure 5, we can see that the value $\frac{R_{S}}{R_{0}}$ is constant with the air (dark blue line), when the sensor operates in the fresh air, the value of $\frac{R_{S}}{R_{0}} \approx 3.6$, so:

$$
R_{0} \approx \frac{R_{S}}{3.6}
$$

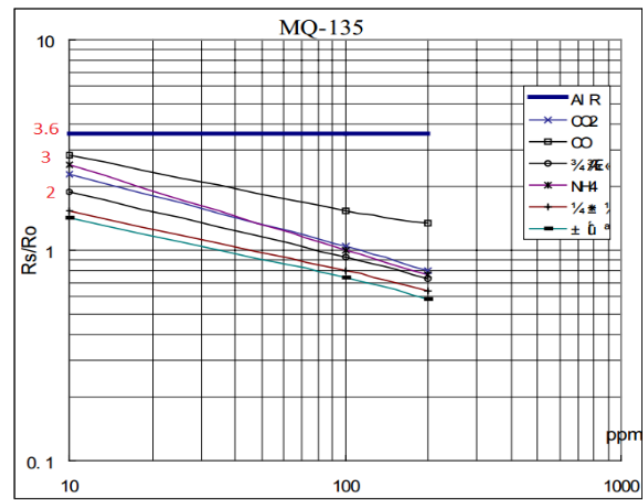

Fig. 5. Find $\mathrm{R}_{0}$

Set the sensor in a room with conditions: clean air, temperature $20^{\circ} \mathrm{C}$, humidity $65 \%, \mathrm{O}_{2} 21 \%$ and $R_{L}=20 \mathrm{k} \Omega$. We have:

$$
R_{S}=\left(\frac{V_{C}}{V_{\text {out }}}-1\right) R_{L}
$$

Replace (4) into (3), we get

$$
R_{0}=\frac{\left(\frac{V_{C}}{V_{\text {out }}}-1\right) R_{L}}{3.6}
$$

where $V_{C}=5 \mathrm{~V}$ and $R_{L}=20 \mathrm{k} \Omega, V_{\text {out }}$ is the output voltage of sensor. We used a program on Arduino IDE for determining $R_{0}$. The result is shown in Figure 6. 


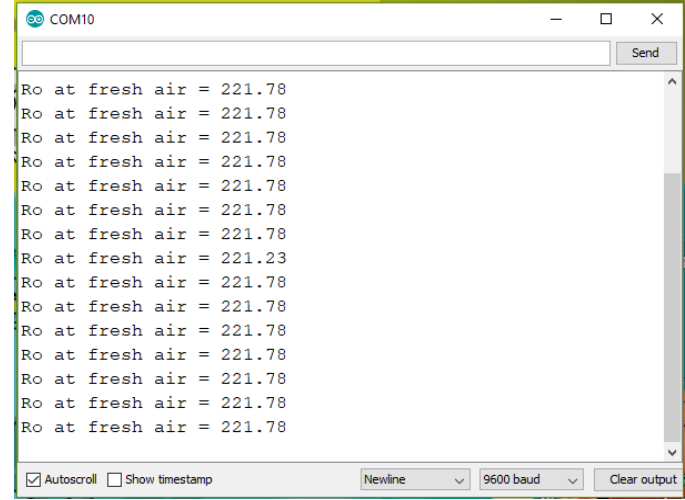

Fig. 6. The stable value of $\mathrm{R}_{0}$ after about 1 hour

After waiting for a stable $R_{0}$ value, we take $R_{0}=222 \mathrm{k} \Omega$. In the next step, we find the relation expression between ppm and $\frac{R_{S}}{R_{0}}$.

The relation between $\log (\mathrm{ppm})$ and $\log \left(\frac{R_{S}}{R_{0}}\right)$ is a linear relationship as represented by the following expression:

$$
\log (y)=m^{*} \log (x)+b
$$

In which $y=\frac{R_{S}}{R_{0}}$ and $x=p p m$. By visualizing through Figure 7, we can see the graph going through 2 points $(10,2.3)$ and $(100,1)$. Thus, by entering (6), we get:

$$
\begin{aligned}
& \Rightarrow\left\{\begin{array}{l}
\log \left(y_{1}\right)=m^{*} \log \left(x_{1}\right)+b \\
\log \left(y_{2}\right)=m^{*} \log \left(x_{2}\right)+b
\end{array}\right. \\
& \Leftrightarrow\left\{\begin{array}{c}
m=-0.362 \\
b=0.724
\end{array}\right.
\end{aligned}
$$

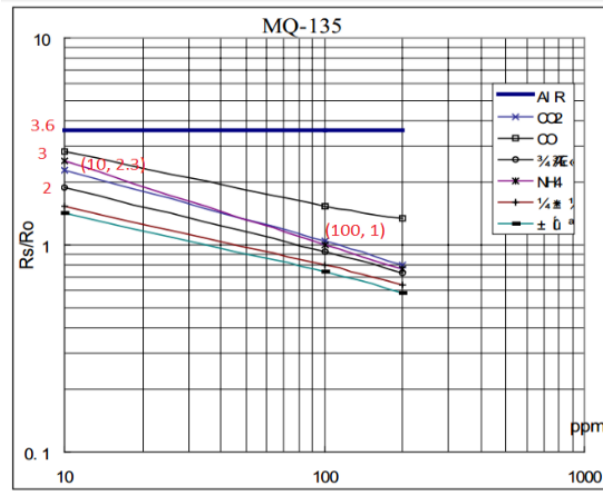

Fig. 7. Find the relationship between ppm and $\frac{R_{S}}{R_{0}}$

$$
\Rightarrow p p m=10^{\left\{\frac{\log \left(\frac{R_{S}}{R_{0}}\right)-b}{m}\right\}}
$$

3. Design Equipment for Measuring and Monitoring Ammonia Concentration

In this section, we present the detail of the developed system used for measuring and monitoring ammonia. In particular, we first introduce the hardware of the system, then design of the software for the system is also presented.

\subsection{Hardware Design}

The schema block of hardware of the developed system is presented in Figure 8. The system consists of some blocks as Source Power, Microcontroller, Wifi module, LCD screen.

\subsubsection{Source Power}

The source is built based on two ICs:

- LM1117 creates 3.3V power feeding ESP8266 and LCD.

- LM1117 5V power supply feeding MQ135, ATMEGA328P.

\subsubsection{ATMEGA328P Microcontroller}

ATmega328P has a full name: ATmega328P-PU, it belongs to MegaAVR family. ATmega328P is 8 bits microcontroller, based on RISC architecture, 32KB ISP flash memory program that can write and erase thousands of times, 1KB EEPROM, and 2KB SRAM.

With 23 pins can be used for I/O connections, 32 registers, 3 timer/counter programmable, internal and external interrupts (2 commands on an interrupt vector), transfer protocol USART, SPI, I2C serial communication. In addition, an 8-bit analog digital converter (ADC) can be used, programmable watchdog timer, operates with 5 power modes, can use up to 6 pulse width modulation channels (PWM).

\subsubsection{WiFi Module}

ESP8266 is a microprocessor designed by Espressif System. Features of ESP8266 are built-in Wi-Fi. There are many types of boards designed using ESP8266 processor. The difference between board types is the number of GPIOs (GPIOs are the ports used for input or output).

\subsubsection{LCD Screen}

Nokia LCD 5110 is used as LCD screen of the proposed system. The technical parameters of LCD screen are presented in Table 2. 


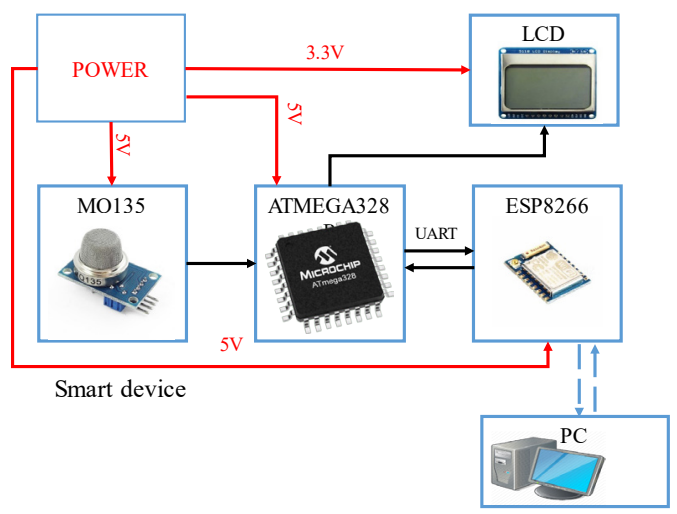

Fig. 8. Block diagram of the device

Table 2. LCD 5110 Specifications

\begin{tabular}{|c|c|}
\hline Operating voltage & $2.7-3.3 \mathrm{VDC}$ \\
\hline Current consumption & $6 \mathrm{~mA}$ \\
\hline Screen size & $\begin{array}{c}84 \times 48 \text { pixel } \\
\text { monochrome }\end{array}$ \\
\hline Communication standard & SPI \\
\hline Processor & Philips PCD8544 \\
\hline Backlight & Blue \\
\hline
\end{tabular}

\section{Software Design}

\subsection{Software for Microcontroller}

\subsubsection{Software for ESP8266}

The flow chart of the program in ESP8266 is presented in Figure 9. We used the $\mathrm{C} / \mathrm{C}++$ language, Arduino as ISP compiler.

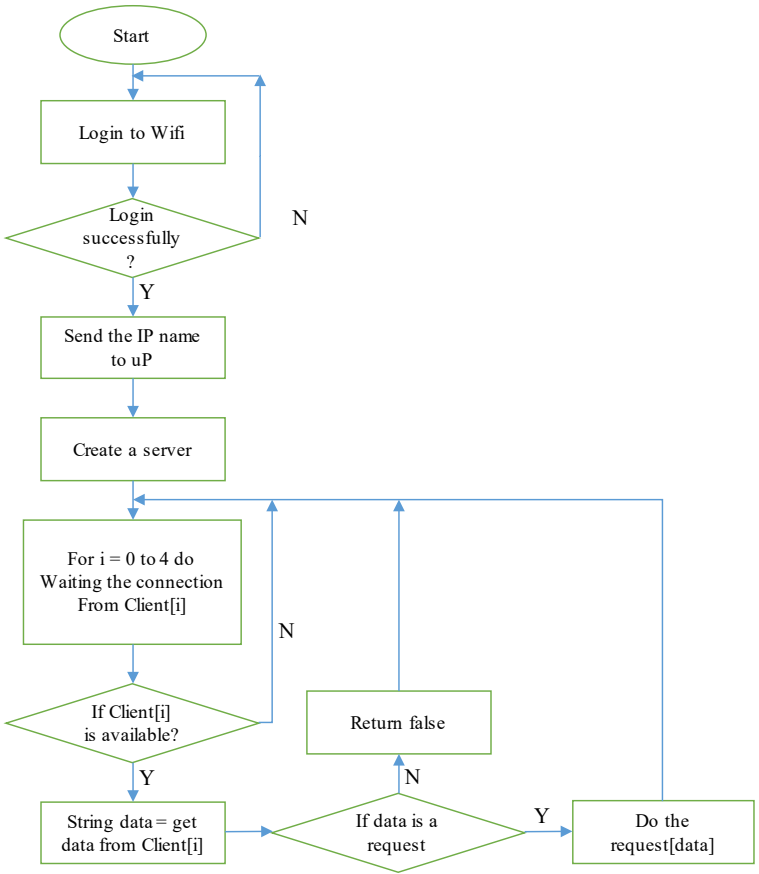

Fig. 9. Algorithm flowchart on ESP8266

\subsubsection{Software for ATMEGA328P}

The flow chart of the software program in MCU ATMEGA328P is presented in Figure 10. We also used use $\mathrm{C} / \mathrm{C}++$ language, Arduino as ISP compiler.

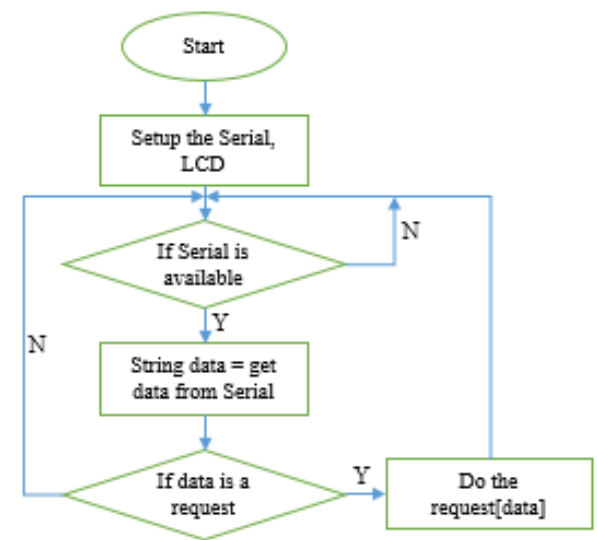

Fig. 10. Algorithm flowchart on ATMEGA328P

\subsection{Software on Computers}

We have been developed a software program based on the Visual Studio C \#. Main functions of this software are listed as follows:

- Connect to the smart device.

- Display and warning measurement results on observation graphs.

- Store the measurement results into the database.

\section{Experimental Results}

The central microcontroller of the measuring device performs the function of reading and processing the measured data from the ammonia gas concentration sensor. The data is then taken to the Communication Module. The device hardware is shown in Figure 11.

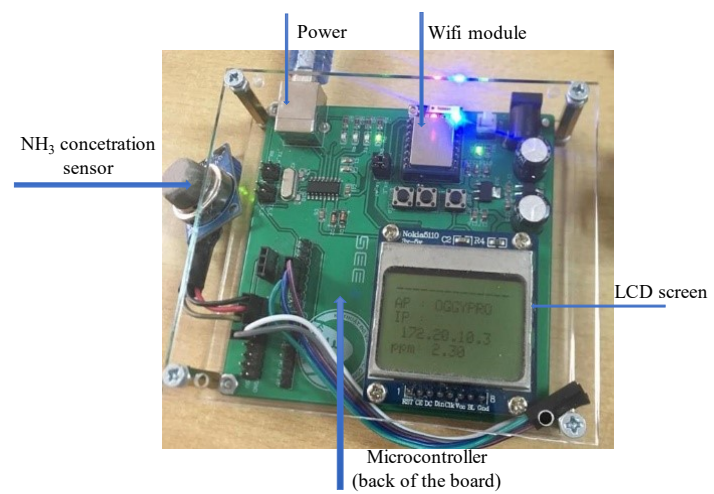

Fig. 11. Hardware board

The communication part from the measurement device to the computer is used via the Wi-Fi module ESP8266. The Graphical User Interface (GUI) is developed base on Visual Studio C \#. The results 
obtained from the device are shown in Figure 12. The evolution of ammonia concentration in the laboratory has fluctuated slightly. Our initial calibration is based on the characteristic curve provided by the sensor characteristic. We, of course, need to calibrate this device in a professional calibration unit.

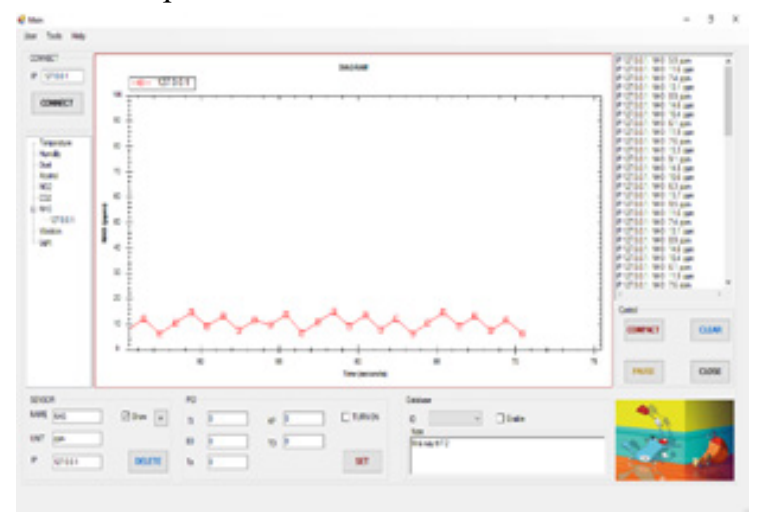

Fig. 12. Results observed in the laboratory

In the current study, we have also used the developed device to test the ambient air environment at NPK company-Lafchemco, Vietnam. The difference in measurement results in this field can be seen with the results in Figure 13. The concentration of ammonia gas doubled the value in the laboratory. However, when compared to the Vietnamese standard, the ammonia concentration in the factory is still within acceptable levels. We believe that the measurement and monitoring of ammonia concentration in the plant should be carried out continuously and permanently to ensure the measurement and warning of exceeding the permissible threshold of ammonia gas concentration. In case the measured value exceeds the allowable thresholds, the system will issue warning messages to the user.

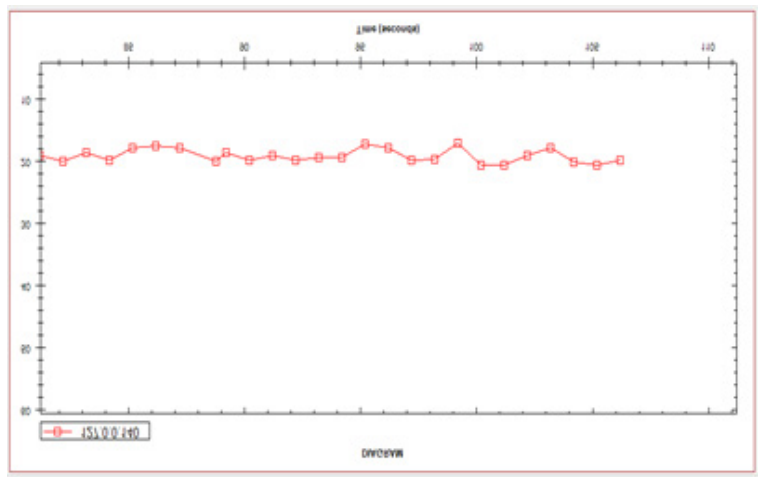

Fig. 13. Measurement results at Lafchemco

\section{Conclusion}

We have presented the development of a device used for ammonia concentration measuring and monitoring. The developed device is built-in Atmega328 microcontroller. The device works well in the laboratory as well as in practical environment with measuring range and monitoring up to $300 \mathrm{ppm}$. The developed algorithms allow the device to smartly alert, implement the communication protocol to the monitoring computer. In addition, the development of connecting devices in IoT (Internet of Things) applications are also the next directions of this study.

\section{Acknowledgment}

This research is funded by Vietnam National Foundation for Science and Technology Development (NAFOSTED) under grant number: 13/2019/TN.

\section{References}

[1] C. G. C.A. Skjøth, The effect of climate and climate change on ammonia emissions in Europe, Atmos. Chem. Phys. 13, pp. 117-128, 2013.

[2] J. W. E. M.A. Sutton, F. Dentener, D. Möller, Ammonia in the environment: from ancient times to the present, Environ. Pollut. 156, pp. 583-604, 2008.

[3] G. K. R. Mani, J.B.B. A highly selective and wide range ammonia sensor-Nanostructured $\mathrm{ZnO}$ : Cothin film.Mater. Sci. Eng. B, 191, pp. 41-50, 2015.

[4] R. N. Ghosh, A.K.; Santra, S.; Pradhan, D.; Guha, P.K. Enhanced ammonia sensing at room temperature with reduced graphene oxide/tin oxide hybrid films.RSC Adv., 5, pp. 50165-50173, 2015.

[5] [A. S. M. Hakimi, and A. Boroumand, Fabrication and characterization of an ammonia gas sensor based on PEDOT-PSS with N-doped graphene quantum dots dopant, IEEE Sensors Journal, Vol.16, No. 16, pp. 6149 - 6154, 2016

[6] A. K. R. Pamula, S. Sai, A. Karnam, and K.J. Suja, Design, simulation and optimization of metal oxide sensor for ammonia detection using nanowires, 2018 International Conference on Emerging Trends and Innovations in Engineering and Technological Research (ICETIETR), Ernakulam, India, pp. 122 $127,2018$.

[7] Z. G. R. P. Gupta, D. Schipanski, and P.D. Vyas, A new room temperature FET-ammonia sensor, 56th Annual Device Research Conference Digest (Cat. No.98TH8373), Charlottesville, VA, USA, pp. 2 - 3, 1998.

[8] P. C. Sun, Y.; Du, S.; Xu, X.; You, L.; Ma, J.; Liu, F.; Liang, X.; Sun, Y.; Lu, G. Hierarchical -Fe2O3/SnO2 semiconductor composites: Hydrothermal synthesis and gas sensing properties. Sens. Actuators B Chem. 182, pp. 336-343, 2013.

[9] Q. L. Feng, X.; Wang, J.; Gaskov, A.M. Reduced graphene oxide (rGO) encapsulated $\mathrm{Co} 3 \mathrm{O} 4$ composite nanofibers for highly selective ammonia sensors. Sens. Actuators B Chem., 222, pp. 864-870, 2016.

[10] K. M. Arshak, E.; Lyons, G.M.; Harris, J.; Clifford, S. A review of gas sensors employed in electronic nose applications. Sens. Rev., 24, pp.181-198, 2004. 
[11] N. J. C. R. Balint, S.H. Cartmell, Conductive polymers: towards a smart biomaterial for tissue engineering, Acta Biomater. 10, pp. 2341-2353, 2014.

[12] J. C. R.S. Andre, D. Kwak, D.S. Correa, L.H.C. Mattoso, Y. Lei, A flexible and disposable poly(sodium 4-styrenesulfonate)/polyaniline coated glass microfiber paper for sensitive and selective detection of ammonia at room temperature, and $\mathrm{p}$. Synth. Met. 233, 2017.

[13] a. P. W. S. Jindarat, Smart farm monitoring using raspberry pi and arduino, 2015 International
Conference on Computer, Communications, and Control Technology (I4CT), Kuching, Malaysia, pp. 21-23, 2015.

[14] https://circuitdigest.com/microcontrollerprojects/arduino-mq137-ammonia-sensor.

[15] G. S. N. Yamazoe, and K. Shimanoe, Oxide semiconductor gas sensors, Catalysis survey from Asia, Vol. 7, No.1, pp. 63-75, 2003. 